\title{
On log-concavity of a class of generalized Stirling numbers
}

\author{
Feng-Zhen Zhao* \\ School of Mathematical Sciences \\ Dalian University of Technology \\ Dalian 116024, China. \\ fengzhenzhao@yahoo.com.cn
}

Submitted: Sep 19, 2011; Accepted: Apr 8, 2012; Published: Apr 16, 2012

2010 Mathematics Subject Classifications: 05A20, 11B73, 11B83

\begin{abstract}
This paper considers the generalized Stirling numbers of the first and second kinds. First, we show that the sequences of the above generalized Stirling numbers are both log-concave under some mild conditions. Then, we show that some polynomials related to the above generalized Stirling numbers are $q$-log-concave or $q$-log-convex under suitable conditions. We further discuss the log-convexity of some linear transformations related to generalized Stirling numbers of the first kind.
\end{abstract}

Keywords: Stirling numbers; log-concavity/log-convexity; $q$-log-concavity/q-logconvexity

\section{Introduction}

For a given sequence $a=\left(a_{0}, a_{1}, \cdots, a_{n}, \cdots\right)$ of real numbers, we let $s_{a}(n, k)$ and $S_{a}(n, k)$ denote the generalized Stirling numbers of the first and second kinds, respectively. That is, $s_{a}(n, k)$ and $S_{a}(n, k)$ are defined respectively by

$$
\sum_{k=0}^{n} s_{a}(n, k) x^{k}=(x \mid a)_{n}
$$

and

$$
\sum_{n=k}^{\infty} S_{a}(n, k) x^{n}=\frac{x^{k}}{\left(1-a_{0} x\right)\left(1-a_{1} x\right) \cdots\left(1-a_{k} x\right)},
$$

*This work was supported in part by the Science Research Foundation of Dalian University of Technology (2008) and the NSFC Grant \# 11061020. 
where

$$
\begin{aligned}
(x \mid a)_{n} & = \begin{cases}\left(x-a_{0}\right)\left(x-a_{1}\right) \cdots\left(x-a_{n-1}\right), & n \geqslant 1 \\
1, & n=0\end{cases} \\
s_{a}(0,0) & =1, \\
s_{a}(n, k) & =0 \text { for } n<k, \\
s_{a}(n, 0) & =(-1)^{n} a_{0} a_{1} \cdots a_{n-1}, \\
S_{a}(n, 0) & =a_{0}^{n} \text { for } n \geqslant 1, \\
S_{a}(n, n) & =1 \text { for } n \geqslant 0 .
\end{aligned}
$$

It is well known that $s_{a}(n, k)$ and $S_{a}(n, k)$ are generalizations of a series of combinatorial numbers. In particular, when $a_{n}=n, s_{a}(n, k)$ and $S_{a}(n, k)$ reduce to the Stirling numbers of the first kind $s(n, k)$ and the second kind $S(n, k)$, respectively. If $a_{n}=-1$, $s_{a}(n, k)$ becomes the binomial coefficient $\left(\begin{array}{l}n \\ k\end{array}\right)$ and, if $a_{n}=1+m n, s_{a}(n, k)$ becomes the Whitney number of the first kind [1]. In addition, when $a_{n}=n^{p}$ or $a_{n}=\left(\begin{array}{c}n+p-1 \\ p\end{array}\right)$ with $p$ to be a nonnegative integer, Sun [14] gave a combinatorial interpretation of $s_{a}(n, k)$ and $S_{a}(n, k)$. For more properties of $s_{a}(n, k)$ and $S_{a}(n, k)$, we refer the reader to $[14,15]$. In this paper, we focus on the log-concavity of $s_{a}(n, k)$ and $S_{a}(n, k)$.

We next recall some definitions and notations involved in this paper.

Definition 1.1. Let $\left\{x_{n}\right\}_{n \geqslant 0}$ be a sequence of nonnegative numbers.

(1) If $x_{j}^{2} \geqslant x_{j-1} x_{j+1}\left(\right.$ or $\left.x_{j}^{2} \leqslant x_{j-1} x_{j+1}\right)$ for each $j \geqslant 1,\left\{x_{n}\right\}_{n \geqslant 0}$ is called log-concave (or log-convex).

(2) If $x_{0} \leqslant x_{1} \leqslant \cdots \leqslant x_{m-1} \leqslant x_{m} \geqslant x_{m+1} \geqslant \cdots$ for some $m,\left\{x_{n}\right\}_{n \geqslant 0}$ is called unimodal, and $m$ is called a mode of the sequence.

Log-concavity and log-convexity are important properties of combinatorial sequences and they have been found many applications in many subjects such as combinatorics, algebra, geometry, probability and statistics; see for instance [2, 8, 13].

Definition 1.2. Let $q$ be an indeterminate and $\left\{f_{n}(q)\right\}_{n \geqslant 0}$ be a sequence of polynomials in $q$. If, for each $n \geqslant 1, f_{n}^{2}(q)-f_{n-1}(q) f_{n+1}(q)\left(\right.$ or $\left.f_{n-1}(q) f_{n+1}(q)-f_{n}^{2}(q)\right)$ has nonnegative coefficients as a polynomial in $q$, we say that $\left\{f_{n}(q)\right\}_{n \geqslant 0}$ is $q$-log-concave (or $q$-log-convex).

The $q$-log-concavity and $q$-log-convexity of polynomials play an important role in proving the log-concavity and log-convexity of combinatorial sequences; see for instance [16]. For the $q$-log-concavity and $q$-log-convexity of classical polynomials, see for instance $[5,6,7,12]$.

Definition 1.3. Let $\{a(n, k)\}_{0 \leqslant k \leqslant n}$ be a triangular array with $a(n, k) \geqslant 0$. 
(1) For a given sequence $\left\{x_{n}\right\}$ of nonnegative numbers, define a linear transformation by

$$
z_{n}=\sum_{k=0}^{n} a(n, k) x_{k}, \quad n=0,1,2, \cdots
$$

If the log-concavity of $\left\{x_{n}\right\}$ implies that of $\left\{z_{n}\right\}$, we say that the linear transformation (1.3) has the preserving log-concavity (PLC) property and the corresponding triangle $\{a(n, k)\}_{0 \leqslant k \leqslant n}$ is called $P L C$.

(2) For a given integer $0 \leqslant r \leqslant n$, let

$$
\mathcal{A}_{r}(n ; q)=\sum_{k=r}^{n} a(n, k) q^{k}
$$

If, for each $r \geqslant 0$, the sequence $\left\{\mathcal{A}_{r}(n ; q)\right\}_{n \geqslant r}$ of polynomials is $q$-log-concave in $n$, we say that the triangle $\{a(n, k)\}_{0 \leqslant k \leqslant n}$ has the $L C$-positive property.

See [16] for more details about the PLC property and the LC-positive property.

Definition 1.4. [2] A sequence of positive numbers whose generating function has only real zeros is called a Pólya frequency sequence (or a PF sequence).

The zeros of the generating function of a finite sequence play an important role in studying the log-concavity of the sequence. A classical approach of proving the logconcavity of a finite sequence is to use the Newton's inequality. In particular, from the Newton's inequality, a PF sequence must be log-concave. See [9] for more information about the PF sequences.

Denote by $c_{a}(n, k)=(-1)^{n+k} s_{a}(n, k)$, which is obviously a generalization of the unsigned Stirling numbers of the first kind. It is well known that the sequence of the unsigned Stirling numbers of the first kind and the sequence of the Stirling numbers of the second kind are both log-concave; see $[3,17]$. The purpose of this paper is to discuss the log-concavity of $c_{a}(n, k)$ and $S_{a}(n, k)$. Meanwhile, we investigate the $q$-log-concavity and $q$-log-convexity of some polynomials related to $c_{a}(n, k)$ and $S_{a}(n, k)$.

Given a sequence $a=\left(a_{0}, a_{1}, \cdots, a_{n}, \cdots\right)$, we define $\langle x \mid a\rangle_{n}$ by

$$
\langle x \mid a\rangle_{n}=\left\{\begin{array}{lr}
\left(x+a_{0}\right)\left(x+a_{1}\right) \cdots\left(x+a_{n-1}\right), & n \geqslant 1, \\
1, & n=0 .
\end{array}\right.
$$

Throughout the paper, we assume that $a=\left(a_{0}, a_{1}, \cdots, a_{n}, \cdots\right)$ is a nonnegative sequence.

\section{Log-concavity of $c_{a}(n, k)$ and $S_{a}(n, k)$}

Recall that the Stirling numbers of two kinds, $(-1)^{n+k} s(n, k)$ and $S(n, k)$, are both $\log$ concave. We discuss the log-concavity of $c_{a}(n, k)$ and $S_{a}(n, k)$ in this section. To this end, we first give some lemmas. 
Lemma 2.1 (Newton's inequality). [10] If the polynomial $b_{0}+b_{1} x+b_{2} x^{2}+\cdots+b_{n} x^{n}$ has only real roots, then

$$
b_{k}^{2} \geqslant b_{k+1} b_{k-1} \frac{k(n-k+1)}{(k-1)(n-k)}
$$

for each $2 \leqslant k \leqslant n-1$.

Lemma 2.2. [2] Let $\left\{b_{k}\right\}_{0 \leqslant k \leqslant n}$ be a sequence of positive real numbers such that the polynomial $\sum_{k=0}^{n} b_{k} x^{k}$ has only real roots, that is, $\left\{b_{k}\right\}_{0 \leqslant k \leqslant n}$ is a PF sequence. Then every mode $k_{0}$ of the sequence $\left\{b_{k}\right\}_{0 \leqslant k \leqslant n}$ satisfies

$$
\left\lfloor\frac{\sum_{k=0}^{n} k b_{k}}{\sum_{k=0}^{n} b_{k}}\right\rfloor \leqslant k_{0} \leqslant\left\lceil\frac{\sum_{k=0}^{n} k b_{k}}{\sum_{k=0}^{n} b_{k}}\right\rceil,
$$

where $\lfloor x\rfloor$ and $\lceil x\rceil$ denote the floor and ceiling of $x$, respectively.

By (1.1), we have

$$
\begin{aligned}
\sum_{k=0}^{n} c_{a}(n, k) x^{k} & =\langle x \mid a\rangle_{n}, \\
c_{a}(n, k) & =c_{a}(n-1, k-1)+a_{n-1} c_{a}(n-1, k), \quad n \geqslant k, k \geqslant 1,
\end{aligned}
$$

and, by $(2.2)$, we can derive $c_{a}(n, k)=\left|s_{a}(n, k)\right|$. Therefore, from (2.1) and Lemma 2.1, we know that $\left\{c_{a}(n, k)\right\}_{0 \leqslant k \leqslant n}$ is log-concave and hence it is unimodal. We further have the following result.

Theorem 2.3. Assume that the sequence $a=\left(a_{0}, a_{1}, \cdots, a_{n}, \cdots\right)$ satisfies $a_{n}>0$ for each $n \geqslant 0$. Then every mode $k_{0}$ of the sequence $\left\{c_{a}(n, k)\right\}_{0 \leqslant k \leqslant n}$ satisfies

$$
\left\lfloor\sum_{j=0}^{n-1} \frac{1}{a_{j}}\right\rfloor \leqslant k_{0} \leqslant\left\lceil\sum_{j=0}^{n-1} \frac{1}{a_{j}}\right\rceil
$$

for each $n \geqslant 2$.

Proof. Let $P(x)=\sum_{k=0}^{n} c_{a}(n, k) x^{k}$. It follows that $P(x)=\langle x \mid a\rangle_{n}$. Making use of Lemma 2.2 , we have

$$
\left\lfloor\frac{\sum_{k=0}^{n} k c_{a}(n, k)}{\sum_{k=0}^{n} c_{a}(n, k)}\right\rfloor \leqslant k_{0} \leqslant\left\lceil\frac{\sum_{k=0}^{n} k c_{a}(n, k)}{\sum_{k=0}^{n} c_{a}(n, k)}\right\rceil .
$$

On the other hand, we have

$$
\frac{P^{\prime}(1)}{P(1)}=\frac{\sum_{k=0}^{n} k c_{a}(n, k)}{\sum_{k=0}^{n} c_{a}(n, k)}=\sum_{j=0}^{n-1} \frac{1}{a_{j}} .
$$

The conclusion follows immediately. 
The following lemma will be useful later on.

Lemma 2.4. [16] The constant triangle $\{a(n, k)\}$ is LC-positive and any LC-positive triangle must be PLC.

Theorem 2.5. Assume that $a=\left(a_{0}, a_{1}, \cdots, a_{n}, \cdots\right)$ is a sequence of nonnegative real numbers such that $a_{0}=0, a_{i} \neq a_{j}$ for $i \neq j$, and $a_{j}>0$ for $j \geqslant 1$. For any fixed $k \geqslant 2$, $\left\{S_{a}(n, k)\right\}_{n \geqslant k}$ is log-concave in $n$.

Proof. We first consider the case where $k=2$. we have from (1.2) that

$$
S_{a}(n, 2)=\frac{a_{2}^{n-1}-a_{1}^{n-1}}{a_{2}-a_{1}}
$$

for $n \geqslant 2$ and, for $n \geqslant 3$, we have

$$
S_{a}^{2}(n, 2)-S_{a}(n-1,2) S_{a}(n+1,2)=a_{1}^{n-1} a_{2}^{n-1} \geqslant 0 .
$$

This means that $\left\{S_{a}(n, 2)\right\}$ is log-concave.

Suppose that $\left\{S_{a}(n, k)\right\}$ is log-concave in $n$ when $k \geqslant 3$. It is sufficient to show that $\left\{S_{a}(n+k+1, k+1)\right\}$ is log-concave in $n$. In fact, by (1.2), we have

$$
S_{a}(n+k+1, k+1)=\sum_{j=0}^{n} S_{a}(j+k, k) a_{k+1}^{n-j}
$$

for $n \geqslant 0$. Noting that $\left\{S_{a}(j+k, k) a_{k}^{-j}\right\}$ is log-concave in $n$, we have from Lemma 2.4 that $\left\{S_{a}(n+k+1, k+1)\right\}$ is log-concave in $n$. This completes the proof.

\section{$3 q$-Log-concavity and $q$-Log-convexity of Some Poly- nomials}

Now we discuss the $q$-log-convexity or $q$-log-concavity of some polynomials related to $s_{a}(n, k)$ and $S_{a}(n, k)$.

Theorem 3.1. Consider the polynomials

$$
F_{n, 1}(q)=\sum_{k=0}^{n} c_{a}(n, k) q^{k}
$$

and

$$
G_{n, 1}(q)=F_{n, 1}^{2}(q)-F_{n-1,1}(q) F_{n+1,1}(q), \quad n \geqslant 1 .
$$

Then we have the following statements: 
(i) $\left\{F_{n, 1}(q)\right\}_{n \geqslant 0}$ is q-log-concave if $a=\left(a_{0}, a_{1}, \cdots, a_{n}, \cdots\right)$ is monotonic decreasing. Conversely, $\left\{F_{n, 1}(q)\right\}_{n \geqslant 0}$ is q-log-convex if $a=\left(a_{0}, a_{1}, \cdots, a_{n}, \cdots\right)$ is monotonic increasing.

(ii) $\left\{G_{n, 1}(q)\right\}_{n \geqslant 1}$ is $q$-log-convex if $a=\left(a_{0}, a_{1}, \cdots, a_{n}, \cdots\right)$ is monotonic increasing and $\left\{a_{n}-a_{n-1}\right\}_{n \geqslant 1}$ is log-convex. Conversely, $\left\{G_{n, 1}(q)\right\}_{n \geqslant 1}$ is q-log-concave if $a=\left(a_{0}, a_{1}, \cdots, a_{n}, \cdots\right)$ is monotonic decreasing and $\left\{a_{n-1}-a_{n}\right\}_{n \geqslant 1}$ is log-concave.

Proof. It is evident that

$$
F_{n, 1}^{2}(q)-F_{n-1,1}(q) F_{n+1,1}(q)=\langle q \mid a\rangle_{n-1}\langle q \mid a\rangle_{n}\left(a_{n-1}-a_{n}\right)
$$

and the coefficients of $\langle q \mid a\rangle_{n}$ are all nonnegative for $n \geqslant 0$. Therefore, $\left\{F_{n, 1}(q)\right\}_{n \geqslant 0}$ is $q$-log-concave when $a=\left(a_{0}, a_{1}, \cdots, a_{n}, \cdots\right)$ is monotonic decreasing and, equivalently, $\left\{F_{n, 1}(q)\right\}_{n \geqslant 0}$ is $q$-log-convex when $a=\left(a_{0}, a_{1}, \cdots, a_{n}, \cdots\right)$ is monotonic increasing.

On the other hand, it is not difficult to see that

$$
\begin{aligned}
& G_{n, 1}^{2}(q)-G_{n-1,1}(q) G_{n+1,1}(q) \\
= & F_{n-2,1}(q) F_{n-1,1}(q) F_{n, 1}^{2}(q)\left[\left(q+a_{n-2}\right)\left(a_{n-1}-a_{n}\right)^{2}\right. \\
& \left.-\left(q+a_{n}\right)\left(a_{n-2}-a_{n-1}\right)\left(a_{n}-a_{n+1}\right)\right] \\
= & F_{n-2,1}(q) F_{n-1,1}(q) F_{n, 1}^{2}(q)\left[\left(a_{n-1}-a_{n}\right)^{2} q+a_{n-2}\left(a_{n-1}-a_{n}\right)^{2}\right. \\
& \left.-\left(a_{n-1}-a_{n-2}\right)\left(a_{n+1}-a_{n}\right) q-a_{n}\left(a_{n-1}-a_{n-2}\right)\left(a_{n+1}-a_{n}\right)\right] .
\end{aligned}
$$

If $a=\left(a_{0}, a_{1}, \cdots, a_{n}, \cdots\right)$ is monotonic increasing and, for $n \geqslant 1$, the sequence $\left\{a_{n}-a_{n-1}\right\}$ is log-convex, we then have

$$
\begin{aligned}
\left(a_{n-1}-a_{n}\right)^{2}-\left(a_{n-1}-a_{n-2}\right)\left(a_{n+1}-a_{n}\right) & \leqslant 0, \\
a_{n-2}\left(a_{n-1}-a_{n}\right)^{2}-a_{n}\left(a_{n-1}-a_{n-2}\right)\left(a_{n+1}-a_{n}\right) & \leqslant 0,
\end{aligned}
$$

which implies that $G_{n-1,1}^{2}(q) G_{n+1,1}^{2}(q)-G_{n, 1}^{2}(q)$ has nonnegative coefficients as a polynomial in $q$ and hence $\left\{G_{n, 1}(q)\right\}_{n \geqslant 1}$ is $q$-log-convex. Conversely, if $a=\left(a_{0}, a_{1}, \cdots, a_{n}, \cdots\right)$ is monotonic decreasing and $\left\{a_{n-1}-a_{n}\right\}_{n \geqslant 1}$ is log-concave, we have

$$
\begin{aligned}
\left(a_{n-1}-a_{n}\right)^{2}-\left(a_{n-1}-a_{n-2}\right)\left(a_{n+1}-a_{n}\right) & \geqslant 0, \\
a_{n-2}\left(a_{n-1}-a_{n}\right)^{2}-a_{n}\left(a_{n-1}-a_{n-2}\right)\left(a_{n+1}-a_{n}\right) & \geqslant 0,
\end{aligned}
$$

which means that $G_{n, 1}^{2}(q)-G_{n-1,1}^{2}(q) G_{n+1,1}^{2}(q)$ has nonnegative coefficients as a polynomial in $q$ and hence $\left\{G_{n, 1}(q)\right\}_{n \geqslant 1}$ is $q$-log-concave.

Theorem 3.2. Assume that the sequence $a=\left(a_{0}, a_{1}, \cdots, a_{n}, \cdots\right)$ satisfies $a_{0}=0, a_{i} \neq a_{j}$ for $i \neq j$, and $a_{j}>0$ for $j \geqslant 1$. Then, for a given $k \geqslant 2,\left\{T_{n, k}(q)\right\}$ is $q$-log-concave, where $T_{n, k}(q)=\sum_{j=0}^{n} S_{a}(j+k, k) q^{j}$. 
Proof. For $n \geqslant 1$, we have

$$
\begin{aligned}
& T_{n, k}^{2}(q)-T_{n-1, k}(q) T_{n+1, k}(q) \\
& =\left[S_{a}(n+k, k) q^{n}-S_{a}(n+k+1, k) q^{n+1}\right] T_{n, k}(q)+S_{a}(n+k, k) S_{a}(n+k+1, k) q^{2 n+1} \\
& =S_{a}(n+k, k) q^{n}+\sum_{j=1}^{n}\left[S_{a}(n+k, k) S_{a}(j+k, k)-S_{a}(n+k+1, k) S_{a}(j-1+k, k)\right] q^{n+j} .
\end{aligned}
$$

Noting that $\left\{S_{a}(n, k)\right\}$ is log-concave in $n$, we have

$$
\frac{S_{a}(n+k, k)}{S_{a}(n+k+1, k)} \geqslant \frac{S_{a}(j-1+k, k)}{S_{a}(j+k, k)} \quad(1 \leqslant j \leqslant n),
$$

that is, $S_{a}(n+k, k) S_{a}(j+k, k)-S_{a}(n+k+1, k) S_{a}(j-1+k, k) \geqslant 0(1 \leqslant j \leqslant n)$. Hence $\left\{T_{n, k}(q)\right\}$ is $q$-log-concave.

\section{Log-convexity of Linear Transformations Related to $c_{a}(n, k)$}

In this section, we discuss the log-convexity of some linear transformations related to $c_{a}(n, k)$. If the sequence $\left\{x_{k}\right\}$ of positive real numbers is log-convex, Liu and Wang [11] proved that $\sum_{k=0}^{n} c(n, k) x_{k}$ preserves the log-convexity, where $c(n, k)$ is the signless Stirling number of the first kind. Now we discuss the log-convexity of $z_{n}=\sum_{k=0}^{n} c_{a}(n, k) x_{k}$.

Theorem 4.1. Suppose that $a=\left(a_{0}, a_{1}, \cdots, a_{n}, \cdots\right)$ satisfies $a_{n+1}-a_{n} \geqslant 1$ for $n \geqslant 0$. If $\left\{x_{n}\right\}_{n \geqslant 0}$ is log-convex and monotonic decreasing, then $\left\{z_{n}=\sum_{k=0}^{n} c_{a}(n, k) x_{k}\right\}$ is $\log _{\text {- }}$ convex for $n \geqslant 2$.

Proof. For $n \geqslant 2$, it follows from (2.2) that

$$
\begin{aligned}
z_{n}^{2}-z_{n-1} z_{n+1}= & z_{n}^{2}-a_{n} z_{n-1} z_{n}-z_{n-1} \sum_{k=1}^{n+1} c_{a}(n, k-1) x_{k} \\
= & z_{n}\left[a_{n-1} z_{n-1}+\sum_{k=1}^{n} c_{a}(n-1, k-1) x_{k}-a_{n} z_{n-1}\right]-z_{n-1} \sum_{k=1}^{n+1} c_{a}(n, k-1) x_{k} \\
= & \left(a_{n-1}-a_{n}\right) z_{n-1} z_{n}+z_{n} \sum_{k=0}^{n-1} c_{a}(n-1, k) x_{k} \\
& +z_{n} \sum_{k=0}^{n-1} c_{a}(n-1, k)\left(x_{k+1}-x_{k}\right)-z_{n-1} \sum_{k=1}^{n+1} c_{a}(n, k-1) x_{k} .
\end{aligned}
$$

Noting that $a_{n-1}-a_{n} \leqslant-1$ and $\left\{x_{n}\right\}_{n \geqslant 0}$ is monotonic decreasing, we have $z_{n}^{2}-z_{n-1} z_{n+1} \leqslant$ 0 , which indicates that $\left\{z_{n}\right\}$ is log-convex for $n \geqslant 2$. 
Theorem 4.2. Suppose that $a=\left(a_{0}, a_{1}, \cdots, a_{n}, \cdots\right)$ satisfies $a_{n+1}-a_{n} \geqslant 1$ for $n \geqslant 0$ and $a_{0}=0$. Then $\left\{z_{n}=\sum_{k=0}^{n} c_{a}(n, k) k\right\}$ is log-convex for $n \geqslant 2$.

Proof. It follows from (2.1) that

$$
\begin{aligned}
& z_{n}^{2}-z_{n-1} z_{n+1} \\
& =\left(\langle 1 \mid a\rangle_{n}\right)^{2}\left(\sum_{j=0}^{n-1} \frac{1}{1+a_{j}}\right)^{2}-\langle 1 \mid a\rangle_{n-1}\langle 1 \mid a\rangle_{n+1} \sum_{j=0}^{n-2} \frac{1}{1+a_{j}} \sum_{j=0}^{n} \frac{1}{1+a_{j}} \\
& =\langle 1 \mid a\rangle_{n-1}\langle 1 \mid a\rangle_{n}\left[\left(a_{n-1}-a_{n}\right)\left(\sum_{j=0}^{n-1} \frac{1}{1+a_{j}}\right)^{2}+\frac{a_{n}-a_{n-1}}{1+a_{n-1}} \sum_{j=0}^{n-1} \frac{1}{1+a_{j}}+\frac{1}{1+a_{n-1}}\right] .
\end{aligned}
$$

Let

$$
g_{n}=\left(a_{n-1}-a_{n}\right)\left(\sum_{j=0}^{n-1} \frac{1}{1+a_{j}}\right)^{2}+\frac{a_{n}-a_{n-1}}{1+a_{n-1}} \sum_{j=0}^{n-1} \frac{1}{1+a_{j}}+\frac{1}{1+a_{n-1}} .
$$

It is sufficient to show by induction that $g_{n} \leqslant 0$ for $n \geqslant 2$. In fact, since $a_{2}-a_{1} \geqslant 1$ and $a_{0}=0$, we have

$$
\begin{aligned}
g_{2} & =\left(a_{1}-a_{2}\right)\left(1+\frac{1}{1+a_{1}}\right)^{2}+\frac{a_{2}-a_{1}}{1+a_{1}}\left(1+\frac{1}{1+a_{1}}\right)+\frac{1}{1+a_{1}} \\
& =a_{1}-a_{2}+\frac{a_{1}-a_{2}}{1+a_{1}}+\frac{1}{1+a_{1}} \\
& <0 .
\end{aligned}
$$

Assume that $g_{n} \leqslant 0$ for $n \geqslant 3$. Since

$$
\begin{aligned}
g_{n+1}= & \left(a_{n}-a_{n+1}\right)\left(\sum_{j=0}^{n-1} \frac{1}{1+a_{j}}+\frac{1}{1+a_{n}}\right)^{2}+\frac{a_{n+1}-a_{n}}{1+a_{n}}\left(\sum_{j=0}^{n-1} \frac{1}{1+a_{j}}+\frac{1}{1+a_{n}}\right) \\
& +\frac{1}{1+a_{n}},
\end{aligned}
$$

by straightforward calculus, we have

$$
\begin{aligned}
g_{n+1} & =\left(a_{n}-a_{n+1}\right)\left(\sum_{j=0}^{n-1} \frac{1}{1+a_{j}}\right)^{2}+\frac{a_{n}-a_{n+1}}{1+a_{n}} \sum_{j=0}^{n-1} \frac{1}{1+a_{j}}+\frac{1}{1+a_{n}} \\
& \leqslant a_{n}-a_{n+1}+\frac{a_{n}-a_{n+1}}{1+a_{n}} \sum_{j=0}^{n-1} \frac{1}{1+a_{j}}+1 \\
& <0 .
\end{aligned}
$$

This completes the proof. 


\section{Conclusions}

We have obtained some properties related to the generalized Stirling numbers of the first and second kinds. In the next step, we will focus on the higher order log-concavity/logconvexity [4] of various combinatorial sequences and, in addition, we will also study the asymptotic approximations of various combinatorial sums.

\section{Acknowledgements}

The author is thankful to an anonymous referee for his/her helpful suggestions and comments.

\section{References}

[1] M. Benoumhani. On Whitney numbers of Dowling lattics. Discrete Mathematics, 159: 13-33, 1996.

[2] F. Brenti. Unimodal, Log-Concave, and Pólya Frequency Sequences in Combinatorics. Memoirs of the American Mathematical Society, 413, 1989.

[3] L. Comtet. Advanced Combinatorics. Reidel Dordrecht, 1974.

[4] W. Y. C. Chen, C. C. Y. Gu, K. J. Ma and L. X. W. Wang. Higher order log-concavity in Euler's difference table. Discrete Mathematics, 311: 2128-2134, 2011.

[5] W. Y. C. Chen, L. X. W. Wang and A. L. B. Yang. Recurrence relations for strongly q-log-convex polynomials. Canadian Mathematical Bulletin, 54: 217-229, 2011.

[6] W. Y. C. Chen, L. X. W. Wang and A. L. B. Yang. Schur positivity and the $q$ log-convexity of the Narayana polynomials. Journal of Algebraic Combinatorics, 32: 303-338, 2010.

[7] W. Y. C. Chen, R. L. Tang, L. X. W. Wang and A. L. B. Yang. The $q$-log-convexity of the Narayana polynomials of type B. Advances in Applied Mathematics, 44: 85-110, 2010.

[8] K. Joag-Dev and F. Proschan. Negative association of random variables with application. The Annals of Statistics, 11: 286-295, 1985.

[9] S. Karlin. Total Positivity, Vol. I. Stanford University Press, 1968.

[10] E. H. Lieb. Concavity properties and generating function for Stirling numbers. Journal of Combinatorial Theory, 5: 203-206, 1968.

[11] L. L. Liu and Y. Wang. On the log-convexity of combinatorial sequences. Advances in Applied Mathematics, 39: 453-476, 2007.

[12] B. E. Sagan. Log-concave sequences of symmetric functions and analogs of the Jaccobi-Trudi determinants. Transactions of the American Mathematical Society, 329: 795-811, 1992. 
[13] R. P. Stanley. Log-concave and unimodal sequences in algebra, combinatorics, and geometry. Annals of the New York Academy of Sciences, 576: 500-535, 1989.

[14] Y. D. Sun. Two classes of $p$-Stirling numbers. Discrete Mathematics, 306: 2801-2805, 2006.

[15] I. Tomescu. Problems in Combinatorics and Graph Theory. A Wiley-Interscience Publication, New York, 1985.

[16] Y. Wang and Y. N. Yeh. Log-concavity and LC-positivity. Journal of Combinatorial Theory, Series A, 114: 195-210, 2007.

[17] H. S. Wilf. Generatingfunctionology. second edition, Academic Press, Boston, MA, 1994. 